\section{CVIA}

CASE REPORT

pISSN 2508-707X / eISSN 2508-7088 https://doi.org/10.22468/cvia.2018.00241 CVIA 2019;3(1):27-29

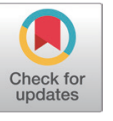

\title{
Correlation Between Functional Myocardial Perfusion Imaging and Anatomical Cardiac CT in a Case of Myocardial Bridging
}

\author{
Mohd Fazrin Mohd Rohani',2, Ahmad Khairuddin Mohamed Yusof ${ }^{3}$, \\ Hazlin Hashim² \\ ${ }^{1}$ Hospital Universiti Sains Malaysia, Kubang Kerian, Malaysia \\ ${ }^{2}$ Advanced Medical and Dental Institute, Universiti Sains Malaysia, Bertam, Malaysia \\ ${ }^{3}$ Institut Jantung Negara, Kuala Lumpur, Malaysia
}

\author{
Received: October 23, 2018 \\ Revised: November 20, 2018 \\ Accepted: November 28, 2018 \\ Corresponding author \\ Mohd Fazrin Mohd Rohani, MD \\ Hospital Universiti Sains Malaysia, \\ Jabatan Perubatan Nuklear, \\ Hospital Universiti Sains Malaysia, \\ Jalan Raja Perempuan Zainab li, \\ 16150, Kubang Kerian, Malaysia \\ Tel: 60-97673486 \\ Fax: 60-97676691 \\ E-mail: fazrinrohani@gmail.com
}

Myocardial perfusion imaging (MPI) with single photon emission computed tomography (SPECT) is generally used to evaluate myocardial ischemia in patients with known or suspected coronary artery disease. Cardiac CT, on the other hand, enables non-invasive visualization of the coronary vessels. Myocardial bridging $(\mathrm{MB})$ is a congenital anomaly characterized by an intramyocardial route of a coronary artery. It is commonly considered benign and rarely requires further evaluation or intervention. However, its clinical complications can be hazardous. We report a patient whose SPECT MPI study demonstrated a mild reversible perfusion defect at the apical anterior segment of the left ventricle that was later attributed to MB visualized on cardiac CT.

Key words Myocardial perfusion imaging · Myocardial bridging Multidetector computed tomography · SPECT.

\section{INTRODUCTION}

Myocardial bridging $(\mathrm{MB})$ is a congenital anomaly characterized by an intramyocardial route of a coronary artery. The coronary arteries tunnel into the myocardium rather than resting on top of it [1]. MB can occur in almost all epicardial arteries; however, the incidence is highest in the left anterior descending artery (LAD) [1]. MB patients are often asymptomatic, and diagnosis is often made from incidental findings of diagnostic imaging, angiography, or autopsy. This congenital anomaly has a good prognosis, with a reported 5-year survival greater than $97 \%$ [2]. However, prognosis can vary from benign to fatal. In symptomatic patients, $\mathrm{MB}$ can present with a variety of clinical manifestations ranging from myocardial ischemia, myocardial infarction, cardiac arrhythmia, to sudden cardiac death [2].

(c) This is an Open Access article distributed under the terms of the Creative Commons Attribution Non-Commercial License (https://creativecommons.org/licenses/by$\mathrm{nc} / 4.0$ ) which permits unrestricted non-commercial use, distribution, and reproduction in any medium, provided the original work is properly cited.

\section{CASE REPORT}

A 56-year-old Indian woman with a history of diabetes mellitus presented with atypical chest pain. Her electrocardiogram (ECG), chest radiograph, cardiac enzymes and other blood parameters were unremarkable. Using the one-day, stress-rest gated Tc-99m tetrofosmin protocol, myocardial perfusion imaging (MPI) was performed for cardiac assessment. Pharmacological stress was applied using dipyridamole infused over 4 minutes at $140 \mu \mathrm{g} / \mathrm{kg} / \mathrm{min}$. There were no symptoms or ECG changes to suggest dipyridamole-induced myocardial ischemia. The myocardial perfusion single photon emission computed tomography (SPECT) images showed mild reversible reduced tracer uptake in the apical anterior segment of the left ventricle (Fig. 1). Other myocardial segments showed no demonstrable perfusion defect. The left ventricular cavity was not dilated.

Subsequently, the patient underwent cardiac CT, which showed a 2-cm-long superficial MB located at the mid segment of the LAD (Figs. 2 and 3). The lumen appeared patent without stenosis. The left circumflex and right coronary arteries were otherwise normal. The coronary calcium score (Agatston 


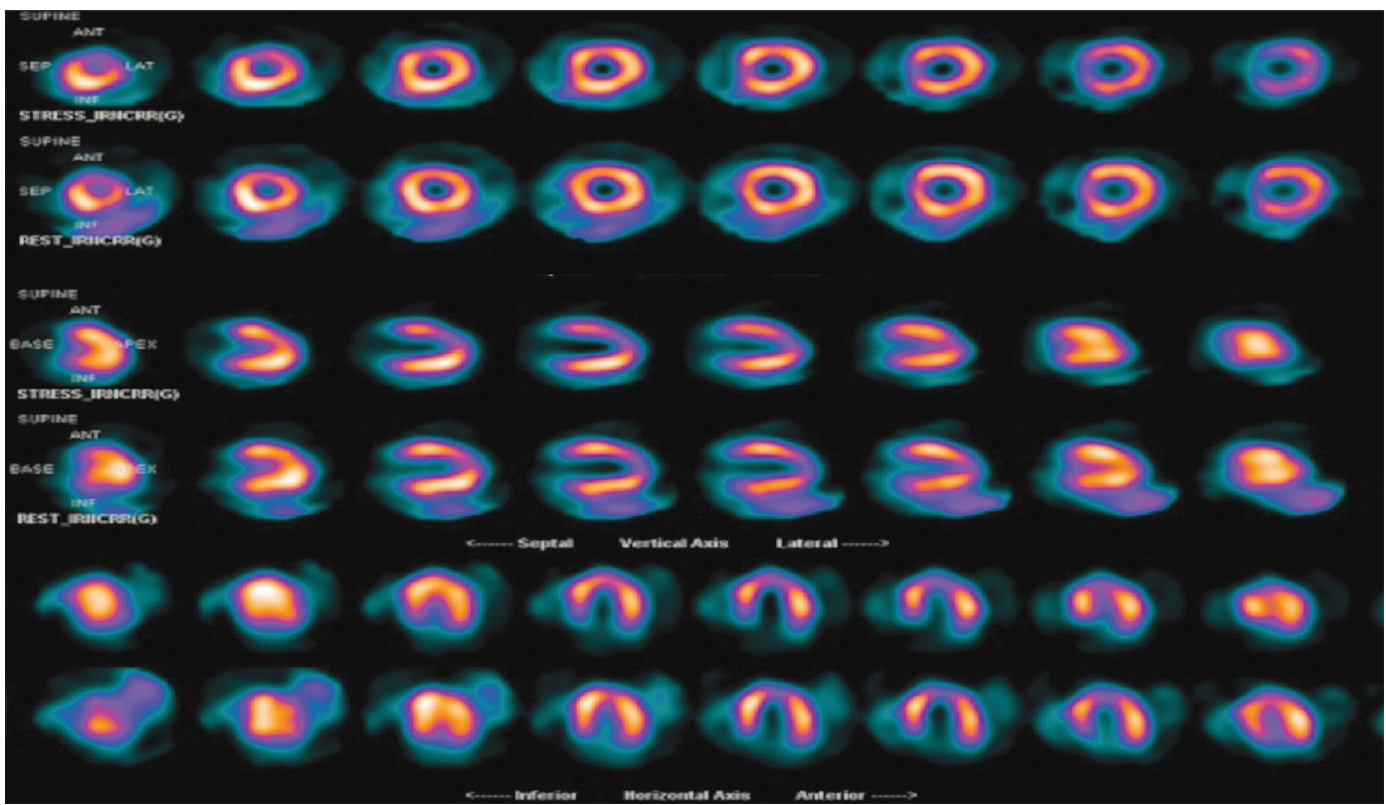

Fig. 1. Patient's stress-rest Tc-99m Tetrofosmin single photon emission computed tomography myocardial perfusion image (from top to bottom); short axis, vertical long axis, horizontal long axis showed mild reversible reduce tracer uptake in the apical anterior segment of left ventricle.

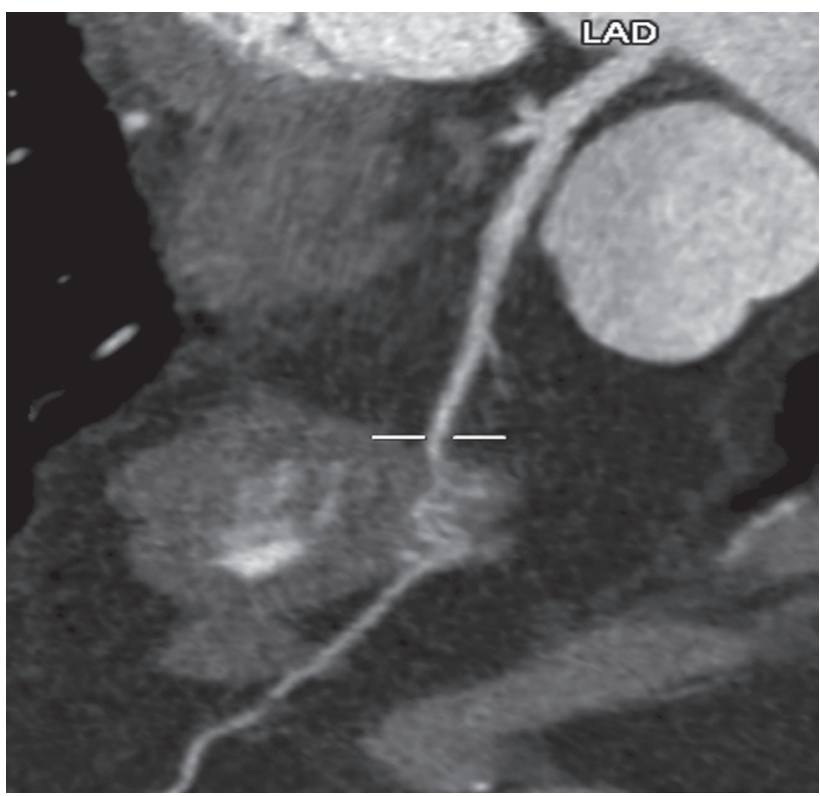

Fig. 2. Cardiac CT showing intramyocardial course of coronary artery at mid segment of LAD. LAD: left anterior descending artery.

score) was 1.2. No coronary arteriography was performed for this patient.

The MPI study showed mild reversible ischemia at the apical anterior segment that was apparently caused by myocardial bridging of the LAD. This case illustrates correlation between functional MPI and anatomical cardiac CT imaging. The patient received medical treatment and follow up in the clinic.

Informed consent was obtained.

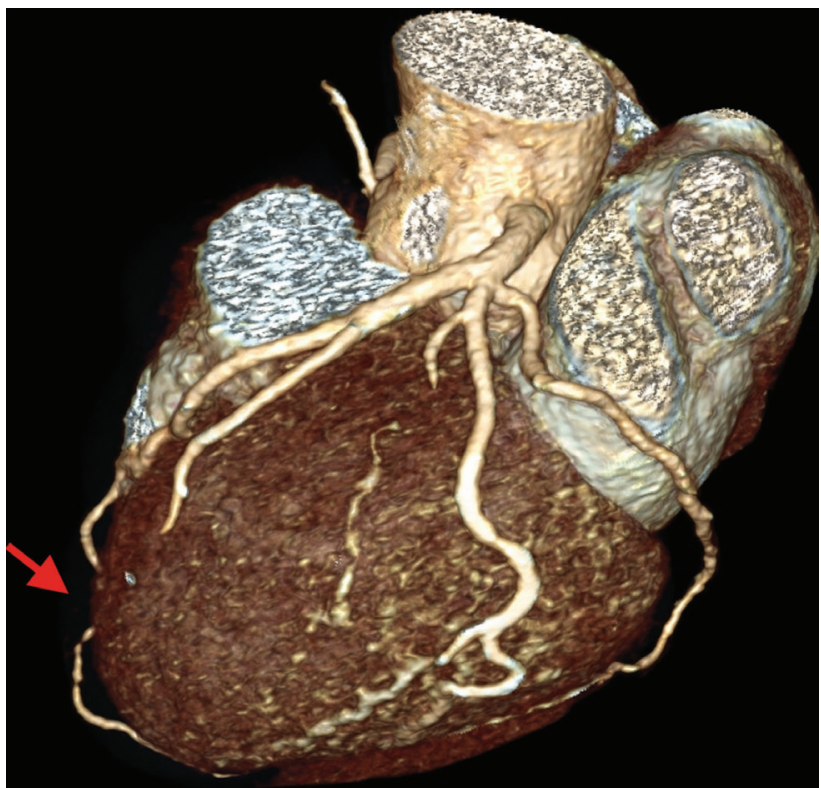

Fig. 3. Volume rendering three-dimensional images which illustrates myocardial bridging (red arrow) at mid segment of the left anterior descending artery.

\section{DISCUSSION}

In patients with $\mathrm{MB}$, the cardiac muscle applies external pressure across the bridged segment during systole, constraining the artery and causing ischemia [3]. The pathophysiology of MB involves a complex interplay between extrinsic and intrinsic factors, including epicardial coronary artery compression, sympathetic drive that prolongs MB contraction, phasic spasm of the coronary artery, an interval interactions among aortic pressure, 
diastolic flow, heart rate, and transmural perfusion gradient $[1,3]$. Additionally, impaired diastolic flow can cause depressurization of the septal branches within the $\mathrm{MB}$, resulting in an intrabridge ischemia, also known as the "branch steal" phenomenon $[1,3]$.

Presently, there are no standardized diagnostic criteria or a gold standard for evaluation of MB [1]. The presence of MB is often noted on anatomical imaging, such as CT scan, which allows characterization of its location, length, and depth; arterial constriction and assessment of atherosclerosis [1,4]. However, the functional significance of MB is often determined by SPECT or positron emission tomography (PET) MPI. Based on the study by Gawor et al. [5] of 42 patients without coronary artery disease, the occurrence of myocardial perfusion defect in bridging patients is linked to the degree of coronary artery narrowing during systole, and patients usually experienced no symptoms if the coronary artery constriction was less than 50\% and were symptomatic if constriction was greater than 70\% [5].

Hybrid cardiac imaging with SPECT/CT [6] and PET/CT [4] allows concurrent evaluation of the coronary vascular anatomy and myocardial blood flow. In a study by Monroy-Gonzalez et al. [4] involving 131 patients who underwent both ${ }^{13} \mathrm{~N}$-ammonia PET and cardiac CT, there was no significant correlation between quantitative perfusion analysis and the anatomical features of MB, such as its length and depth, displayed on CT [4]. Furthermore, myocardial perfusion reserve (MPR), a marker of microvascular dysfunction quantified by PET, was found to be low in patients with MB of the LAD [4]. MPR measured by PET can be used as a tool to identify MB patients who are at increased risk of cardiac event [4].

In practice, a combination of both functional and anatomical imaging can guide the clinician in treatment planning and monitoring of MB [6]. In a case report by Lim et al. [7], a 43year-old man with established $\mathrm{MB}$ refractory to medical treatment underwent a combination of cardiac CT with MPI. The study demonstrated $\mathrm{MB}$ in the mid $\mathrm{LAD}$ and reversible perfusion defect in the anteroseptal wall [7]. The MB was surgically corrected with supraarterial myotomy, and subsequent imaging showed successful unroofing of the MB segment with a normal result on myocardial perfusion study [7].

Treatment is often unnecessary in the asymptomatic MB patient. For symptomatic patients, medical treatment with beta blockers causes reduction in compression by the muscular band, slows the heart rate, and prolongs the diastolic period [1]. Invasive therapy such as percutaneous coronary intervention and coronary artery bypass graft, are usually reserved for severely symptomatic refractory cases and cases that cannot be controlled with oral medications [1,2]. To date, there are no appropriate guidelines on suitable medical treatment or revascularization techniques for MB. Additionally, it is uncertain if intervention in bridging patients with mild or moderate ischemia achieves better outcomes. Thus, there is uncertainty in optimal patient management.

In conclusion, although uncommon, MB should be included in the differential diagnosis for chest pain. A combination of anatomical and functional imaging in patients with $\mathrm{MB}$ provides valuable hemodynamic information on the bridging segment, which guides the clinician's determination of a treatment plan and aids in monitoring of therapy response.

\section{Conflicts of Interest}

The authors declare that they have no conflict of interest.

\section{REFERENCES}

1. Tarantini G, Migliore F, Cademartiri F, Fraccaro C, Iliceto S. Left anterior descending artery myocardial bridging: a clinical approach. J Am Coll Cardiol 2016;68:2887-2899.

2. Ko SM, Choi JS, Nam CW, Hur SH. Incidence and clinical significance of myocardial bridging with ECG-gated 16-row MDCT coronary angiography. Int J Cardiovasc Imaging 2008;24:445-452.

3. Gould KL, Johnson NP. Myocardial bridges: lessons in clinical coronary pathophysiology. JACC Cardiovasc Imaging 2015;8:705-709.

4. Monroy-Gonzalez AG, Alexanderson-Rosas E, Prakken NHJ, JuarezOrozco LE, Walls-Laguarda L, Berrios-Barcenas EA, et al. Myocardial bridging of the left anterior descending coronary artery is associated with reduced myocardial perfusion reserve: a ${ }^{13} \mathrm{~N}$-ammonia PET study. Int J Cardiovasc Imaging 2018 Sep 28 [Epub]. https://doi.org/10.1007/s10554018-1460-8.

5. Gawor R, Kuśmierek J, Płachcińska A, Bieńkiewicz M, Drożdż J, Piotrowski $\mathrm{G}$, et al. Myocardial perfusion GSPECT imaging in patients with myocardial bridging. J Nucl Cardiol 2011;18:1059-1065.

6. Singh D, Vadi SK, Parmar M, Sood A, Mehrotra S, Mittal BR. Role of ${ }^{99 m}$ Tcsestamibi gated SPECT/CT myocardial perfusion imaging in the management of patients with myocardial bridging and its correlation with coronary angiography. J Cardiol Cases 2017;15:136-140.

7. Lim JW, Lee H, Her K, Park HW, Shin KE. Myocardial CT perfusion imaging for pre- and postoperative evaluation of myocardial ischemia in a patient with myocardial bridging: a case report. Medicine (Baltimore) 2017;96:e8277. 